\title{
Red-diode-pumped Cr:Nd:GSGG laser: two-color mode-locked operation
}

\author{
Talha Yerebakan, ${ }^{1}$ Umit Demirbas, ${ }^{1,2,3, *}$ Stefan Eggert, ${ }^{2}$ Rainer Bertram, ${ }^{4}$ Peter Reiche, ${ }^{4}$ and \\ Alfred Leitenstorfer ${ }^{2}$ \\ ${ }^{1}$ Laser Technology Laboratory, Department of Electrical and Electronics Engineering, Antalya International University, 07190 Antalya, Turkey \\ ${ }^{2}$ Department of Physics and Center for Applied Photonics, University of Konstanz, D-78464 Konstanz, Germany \\ ${ }^{3}$ Center for Free-Electron Laser Science, The Hamburg Center of Ultrafast Imaging, Deutsches Elektronen Synchroton (DESY), Hamburg, Germany \\ ${ }^{4}$ Leibniz Institute for Crystal Growth, Max-Born-Str. 2, 12489 Berlin, Germany \\ ${ }^{*}$ Corresponding author: umit79@alum.mit.edu
}

\begin{abstract}
We have investigated in detail the continuous-wave (cw) and mode-locked performance of a diode-pumped Cr:Nd:GSGG laser. State-of-the-art single-mode and multimode laser diodes around $665 \mathrm{~nm}$ were used as pump sources. In cw operation, we have demonstrated lasing thresholds as low as $14 \mathrm{~mW}$, slope efficiencies as high as $23.4 \%$, and output powers up to $738 \mathrm{~mW}$. The free running emission wavelength was $1061 \mathrm{~nm}$. Lasing could also be obtained at 1051, 1058, 1065, 1068, 1072, 1103, and $1111 \mathrm{~nm}$ lines. A saturable Bragg reflector was used to initiate and sustain mode-locking where the Cr:Nd:GSGG laser produced 6-ps-long pulses around $1061 \mathrm{~nm}$ with an average power of $160 \mathrm{~mW}$. The repetition rate was $142.65 \mathrm{MHz}$, resulting in pulse energies of $1.1 \mathrm{~nJ}$ and peak powers of $175 \mathrm{~W}$. An off-surface optical axis quartz birefringent filter (BRF) was inserted inside the laser cavity at Brewster's angle to obtain two-color $\mathrm{cw}$ and mode-locked laser operation at the 1051 and $1058 \mathrm{~nm}$ and 1058 and 1061 transition pairs, resulting in $\mathrm{cw}$ powers up to $60 \mathrm{~mW}$ and $\mathrm{cw}$ mode-locked average powers up to $45 \mathrm{~mW}$. Unlike many other methods applied for two-color mode-locked laser operation, usage of the BRF enabled regulation of the ratio of the power in each line by fine adjustment of its rotation angle. The method could potentially be used for other gain media as well, which could simplify development of multicolor solid-state laser systems. (c) 2017 Optical Society of America
\end{abstract}

\section{INTRODUCTION}

Trivalent neodymium $\left(\mathrm{Nd}^{+3}\right)$-doped yttrium aluminum garnet (YAG) has been known as a versatile gain medium for more than 50 years [1]. It is also well known that flashlamp pumping of $\mathrm{Nd}$ :YAG is not very efficient due to the narrow absorption bands of the $\mathrm{Nd}^{+3}$ ions, which overlap poorly with the broadband flashlamp emitter [2]. Co-doping of Nd:YAG with chromium ions produces a broad absorption band in the visible, and hence improves the absorption efficiency. Unfortunately, the energy transfer process from the chromium ions to the $\mathrm{Nd}$ ions is quite slow in Cr:Nd:YAG (6.2 ms [3]) due to the relatively small lattice parameters of the crystal [4]. As a result, the notion of improving the laser efficiency of $\mathrm{Nd}$ lasers by sensitizing with Cr ions does not work well for Cr:Nd:YAG [4].

The alternative material, $\mathrm{Nd}^{+3}$-doped gadolinium scandium gallium garnet ( $\mathrm{Nd}: \mathrm{Gd}_{3} \mathrm{Sc}_{2} \mathrm{Ga}_{3} \mathrm{O}_{12}$, Nd:GSGG) was first shown to lase by Kaminskii et al. in 1976 [5]. Due to its large lattice parameter, Nd:GSGG co-doped with chromium enables fast $(17 \mu \mathrm{s}[6])$ and efficient (86\% [6]) energy transfer from the $\mathrm{Cr}$ ion to the $\mathrm{Nd}$ ion, thus improving the laser efficiency of the flashlamp-pumped laser systems [5]. It is shown that the flashlamp-pumped Cr:Nd:GSGG system has twice the efficiency of analogous Nd:YAG laser systems [2,7]. Similar performance could also be achieved under solar pumping [8-10].

As an alternative to solar and flashlamp pumping, laser diodes were used for efficient excitation of Cr:Nd:GSGG/ Nd:GSGG lasers [11-13]. Diode pumping enables construction of compact and lower-cost laser systems with improved electrical-to-optical conversion efficiency. Usually, sharp absorption peaks of Nd:GSSG around 808 or $883 \mathrm{~nm}$ are employed for pumping (see $[4,14]$ for an absorption spectrum) [12-16]. On the other hand, besides improving efficiency in flashlamp or solar excitation, co-doping of Nd:GSGG with $\mathrm{Cr}$ ions also allows for red laser diodes as alternative pump sources [11]. 
In this approach, due to the broad absorption band of chromium around $645 \mathrm{~nm}$ with a FWHM of $100 \mathrm{~nm}$, a wide range of pump wavelengths are adequate. Moreover, thermal control of the diode junction temperature, narrowband diode operation, and careful selection of diode central wavelength are not required [11]. Furthermore, in Cr:Nd:GSGG, one has more freedom in optimizing the $\mathrm{Nd}$ concentration of the material for laser efficiency (one can reduce the $\mathrm{Nd}$ concentration to minimize detrimental effects of concentration quenching on lifetime [4], but could still have enough absorption by adjusting the Cr concentration, which can be independently optimized for thermal management [17]). These advantages come at the expense of a higher quantum defect, due to the larger energy difference between the laser and pump photons. In his early work from 1991, Scheps obtained cw output powers as high as $42.8 \mathrm{~mW}$ and a slope efficiency of $22.6 \%$ from a cw $\mathrm{Cr}: \mathrm{Nd}$ : GSGG laser pumped by a $300 \mathrm{~mW}$ multimode diode around $673 \mathrm{~nm}$ [11]. Over the last 25 years, since this initial work, the brightness of the red pump diodes has improved significantly. However, to our knowledge, red-diode pumping of Cr:Nd:GSSG systems has not been studied any further.

A recently evolving active research area in $\mathrm{Nd}$-based laser materials and others is on multicolor lasing. In particular, two-color mode-locked laser operation has been attracting a great deal of attention due to potential usage of such lasers in application like generation of coherent terahertz waves and formation of ultrahigh pulse repetition rates by optical beating. Simultaneous dual/tri-wavelength mode-locked laser operation with picosecond pulses has been shown in many $\mathrm{Nd}$-based laser gain media, including Nd:LYSO [18], Nd:SYSO [19], Nd:LGGG [20-22], Nd:CNGG [23], Nd:CLTGG [24], Nd:GdVO 4 [25], $\mathrm{Nd}: \mathrm{YVO}_{4} / \mathrm{Nd}: \mathrm{GdVO}_{4}$ [26-28], Nd:GAGG [29], Nd:YVO $/$ $\mathrm{Nd}: \mathrm{LuVO}_{4}$ [30], Nd:YVO $/ \mathrm{Nd}: \mathrm{KGW}$ [30], Nd:YSAG [31], $\mathrm{Nd}$ :YAG [32,33], Nd:YAP [34], and Nd:Gd:SrF 2 [35]. In these works, operating in a multiwavelength regime was established using different techniques such as usage of disordered multicenter crystals with inhomogeneously broadened emission spectrum [22], and/or operating with cavity mirrors with specially designed reflectivity properties [33], enrolling two different laser crystals in the cavity [26-28], employment of intracavity etalons [34], and by cooling the laser crystal to cryogenic temperatures [32]. In many of these earlier works, due to the methods used for multicolor laser operation, the ratio of the power in each line varies with external factors such as pump power, crystal temperature and focusing, etc. Most of the time, this results in unequal intensities of the lasing wavelength pair, which might reduce efficiencies in intended application. As an alternative, birefringent tuning plates could also be used for wavelength selection in multicolor lasers. They enable control of the laser line intensities by fine adjustment of their rotation angle. Birefringent tuning plates have been shown to produce dual-wavelength continuouswave laser operation in Ti:sapphire [36,37], Yb:KGW [38], Cr: Nd:GSGG [39], and Cr:LiSAF [39].

In this work, we have investigated laser performance of Cr:Nd:GSGG lasers pumped by state-of-the-art visible laser diodes around $665 \mathrm{~nm}$. In the first set of experiments a $180 \mathrm{~mW}$ low-cost single-mode diode with a TEM $_{00}$ beam profile has been used as the pump source to investigate performance limits of the system. Later, we investigate power scaling with four multimode diodes providing $1.5 \mathrm{~W}$ of pump power each. In cw laser experiments, we have attained output powers up to $738 \mathrm{~mW}$ around $1061 \mathrm{~nm}$. Cw lasing has also been acquired in seven other lines of the $\mathrm{Nd}+3$ ion: 1051, $1058,1065,1068,1072,1103$, and $1111 \mathrm{~nm}$. When mode-locked with a saturable Bragg reflector (SBR [40], also known as semiconductor saturable absorber mirrors [41]), the Cr:Nd:GSGG laser produced 6 ps pulses around $1061 \mathrm{~nm}$, with an average power of $160 \mathrm{~mW}$, using a 1\% output coupler. Moreover, a crystalline quartz birefringent filter (BRF) with an optical axis pointing $45^{\circ}$ out of its surface has been used to obtain dual-color continuous-wave and mode-locked laser operation at 1058-1061 nm and at 1051-1058 nm. In the experiments, usage of the off-surface optical axis BRF enabled adjustment of the laser power in each line by fine adjustment of the rotation angle of the BRF [39]. For the 1058-1061 nm pair, we could achieve a cw output power of $60 \mathrm{~mW}$, and a $\mathrm{cw}$ mode-locked (CWML) average power of $45 \mathrm{~mW}$ using a $0.6 \%$ output coupler. For the 1051-1058 nm pair, due to the lower emission cross-section values, laser power in two-color operation decreased to $35 \mathrm{~mW}$ in the $\mathrm{cw}$ regime, and to $30 \mathrm{~mW}$ in the mode-locked regime, resulting in Q-switched mode-locked (QSML) operation. In future studies, usage of higher-power pump diodes and employment of laser crystals with lower passive losses might enable scaling of output powers, and might facilitate two-color laser operation at many other wavelength pairs. To our knowledge, this is the first demonstration of two-color mode-locked operation with the Cr:Nd:GSGG/Nd:GSGG laser materials, and the first time usage of an off-axis optical surface birefringent filter as a tuning element in multicolor mode-locked lasers. As we have recently discussed in detail in [39], compared to regular BRFs where the optical axis lies in the plane of the plates surface, a BRF with an optical axis pointing out of its surface enables design flexibility in filter parameters, providing access to a wider set of free spectral range and bandwidth values, enabling performance optimization in multicolor laser operation. The method has been demonstrated for Cr:Nd:GSGG in this study; however, it could potentially be used for other gain media as well that could simplify multicolor solid-state laser development efforts.

\section{EXPERIMENTAL}

Figure 1 shows a schematic of the diode-pumped Cr: Nd: GSGG laser setup. The system is pumped either by one single-mode diode (SMD) or by four multimode diodes (MMD-1 to MMD-4). The single-mode diode provided up to $180 \mathrm{~mW}$ of output power around $665 \mathrm{~nm}$. Each multimode diode provided 1.5 W of pump power around $665 \mathrm{~nm}$. All of the diodes were linearly polarized with a polarization extinction ratio better than 100:1. The MMDs had an emitter size of $1 \mu \mathrm{m} \times 150 \mu \mathrm{m}$, with diffraction limited output in the sagittal axis, and an $M^{2}$ of 10 in the tangential axis. Aspheric lenses of focal length of $f=4.5 \mathrm{~mm}$ were used to collimate the output of both the SMDs and MMDs. Two half-wave plates reverse the polarization of MMD-3 and MMD-4. Polarizing beam splitter cubes provided polarization coupling of the pump 


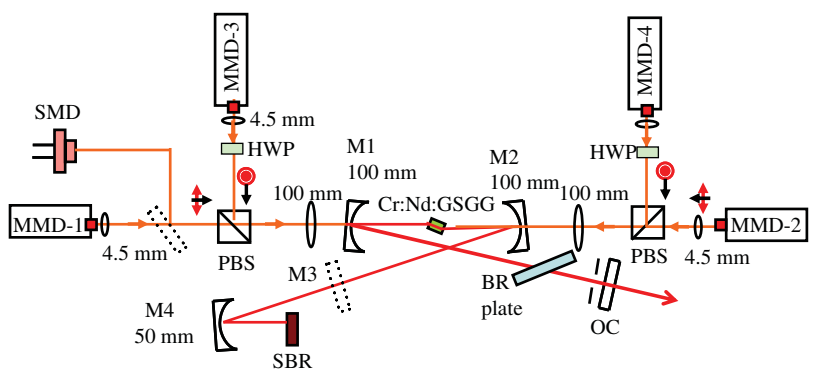

Fig. 1. Schematics of the diode-pumped Cr:Nd:GSGG laser. SMD, single-mode diode; MMD, multimode diode; SBR, semiconductor Bragg reflector; PBS, polarizing beam splitter cube; HWP, half-wave plate; BR plate, off-surface optical axis birefringent tuning plate; OC, output coupler.

beams from each side. Two $100 \mathrm{~mm}$ focal length achromatic doublets were then used to focus the pump beams inside the crystal. The estimated pump beam waists (sagittal $x$ tangential) inside the crystal were $\sim 30 \mu \mathrm{m} \times 130 \mu \mathrm{m}$ and $\sim 30 \mu \mathrm{m} \times$ $60 \mu \mathrm{m}$ for the multimode and single-mode diodes, respectively (beam size is larger in the tangential plane due to the Brewster angle inserted laser crystal).

In the continuous-wave laser experiments, an astigmatically compensated $X$ cavity with two curved pump mirrors (M1 and M2, $R=100 \mathrm{~mm}$ ), a flat end mirror (M3), and an output coupler (OC) were implemented. Arm lengths of $20 \mathrm{~cm}$ $(\mathrm{OC}$ arm) and $50 \mathrm{~cm}$ were used to obtain a laser mode size of $\sim 30 \mu \mathrm{m} \times 60 \mu \mathrm{m}$ inside the Cr:Nd:GSGG crystal. Cavity mirrors (M1-M3) had a reflectivity bandwidth extending from 845 to $1120 \mathrm{~nm}$ and had a transmission of $~ 97 \%$ at the pumping band. The gain medium is a 8.15 long, $0.25 \% \mathrm{Cr}$ - and $0.015 \%$ Nd-doped $\mathrm{Cr}: \mathrm{Nd}: \mathrm{Gd}_{3} \mathrm{Sc}_{2} \mathrm{Ga}_{3} \mathrm{O}_{12}$ crystal. It was grown in-house by the Czochralski method from iridium crucible under nitrogen atmosphere. The melting temperature is about $1700^{\circ} \mathrm{C}$. The growth rate was about $1 \mathrm{~mm} / \mathrm{h}$. To achieve a flat interface the crystal rotation was strongly increased after having reached the cylinder stage. About $2 \%$ of oxygen was added to the nitrogen atmosphere to stabilize $\mathrm{Cr}$ in the +3 charge state. The crystal was $4 \mathrm{~mm}$ thick and mounted with indium foil in a copper holder under water cooling at $15^{\circ} \mathrm{C}$. It was placed at Brewster's angle inside the cavity and absorbed 98.5\% of the incident TM-polarized pump light. The path length of the pump beam inside the crystal at Brewster's configuration was $10.8 \mathrm{~mm}$. For the TE-polarized pump light, $34 \%$ of the incident pump power was lost due to Fresnel reflections, and $98.5 \%$ of the remaining pump power was absorbed by the crystal (overall absorption efficiency was around $65 \%$ for the TE-polarized light). A saturable Bragg reflector with a central reflectivity wavelength of $1040 \mathrm{~nm}$ was used to initiate and sustain mode-locking (BATOP Inc., SAM1040-1-1ps). The modulation depth, the non-saturable loss, the saturation fluence, and the relaxation time of the SBR were specified to be $0.6 \%, 0.4 \%, 70 \mu \mathrm{J} / \mathrm{cm}^{2}$, and $1 \mathrm{ps}$, respectively. The measured reflectivity bandwidth $(\mathrm{R}>99 \%)$ of the SBR extended from 1010 to $1080 \mathrm{~nm}$, enabling flexible laser mode-locked operation in a wide wavelength range. A 3-mmthick crystal quartz birefringent tuning plate (BR plate, or birefringent filter: BRF) with an optical axis pointing $45^{\circ}$ out of the surface plane was inserted into the cavity at Brewster's angle to tune the laser wavelength both in $\mathrm{cw}$ and mode-locked operation. The off-surface optical axis BRF also facilitated two-color $\mathrm{cw}$ and mode-locked laser operation, and its properties will be described in detail in the next section.

\section{RESULTS AND DISCUSSION}

\section{A. Discussion of the Usage of Off-Axis Optical Surface Birefringent Filter for Tuning and Multicolor Laser Operation}

As mentioned in the experimental section, we have used a 3-mm-thick crystal quartz birefringent tuning plate with an optical axis pointing $45^{\circ}$ out of the surface plane in our tuning experiments. This kind of birefringent tuning plate is known as an off-surface optical axis BRF or as a diving angle BRF in the literature. The BRF that was used in this study not only provided tuning in single wavelength operation, but also allowed us to achieve multicolor laser operation. Moreover, it was possible to use the same plate in $\mathrm{cw}$ and mode-locked regimes (with picosecond pulses). Transmission characteristics of such birefringent tuning plates with an arbitrarily oriented optical axis and their advantage in obtaining multicolor laser operation has been recently discussed in [39], and further detailed theoretical information on BRFs can be found in [42-49]. Here we will provide a short description of such BRFs to explain our tuning results that will be presented in the subsequent sections.

As a starting point, Fig. 2 shows the measured emission spectrum of the Cr:Nd:GSGG sample at room temperature around the wavelength region of interest (while being pumped by one of the multimode diodes at $665 \mathrm{~nm}$ ). The positions and strength of the emission peaks match relatively well with the reported spectra in the literature within the uncertainty band given by the resolution bandwidth of our spectrometer (Ocean Optics HR4000, $0.25 \mathrm{~nm}$ ) [4,5]. Note that single wavelength tuning of the laser with such a structured spectrum is relatively

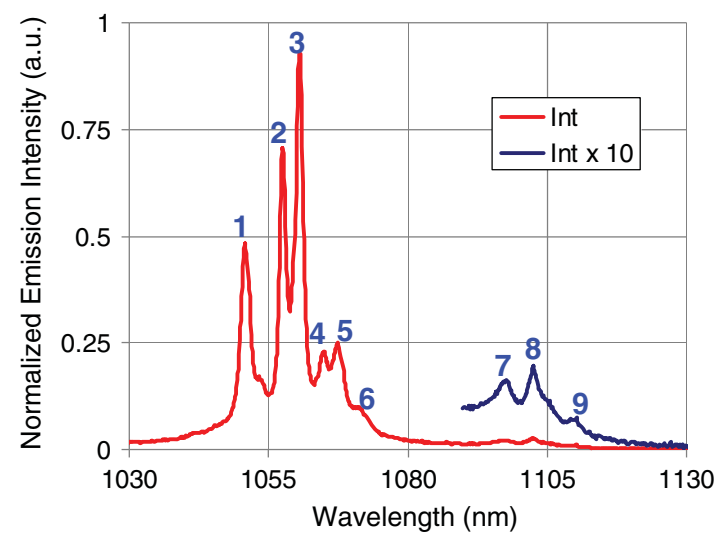

Fig. 2. Measured emission spectrum of the Cr:Nd:GSGG laser at room temperature using a Si-based spectrometer with a resolution of $0.25 \mathrm{~nm}$. Main peak positions are marked by numbers in the graph and correspond to the following wavelengths: $1,1050.9 \mathrm{~nm}$; 2, $1057.6 \mathrm{~nm}$;, $1060.7 \mathrm{~nm}$; $1065.2 \mathrm{~nm} ; 5,1067.7 \mathrm{~nm}$; 6, $1071.9 \mathrm{~nm} ; 7,1098.2 \mathrm{~nm} ; 8,1102.6 \mathrm{~nm} ; 9,1110.3 \mathrm{~nm}$. 
easy, and one just needs to adjust the transmission maxima of the BRF to the desired wavelength, and single wavelength operation is then achieved if the BRF also provides enough suppression at the neighboring transitions (a large enough free spectral range, a sharp enough transmission peak with a low FWHM, and a fast tuning rate are desired, and could be achieved relatively easily).

On the other hand, multiwavelength operation requires equalization of gain at multiple wavelengths at the same time, and is more challenging. As an example, let us assume that we want to obtain two-color mode-locked laser operation at the wavelengths of 1058 and $1061 \mathrm{~nm}$ from the Cr:Nd: GSGG material. Looking at the emission spectrum of $\mathrm{Cr}: \mathrm{Nd}$ : GSGG (Fig. 2), the emission line around $1061 \mathrm{~nm}$ is stronger than the line at $1058 \mathrm{~nm}$. Then we need to provide some loss at $1061 \mathrm{~nm}$ to push the laser to operate at two colors with similar intensities simultaneously (when we roughly equalize the net gain at both transitions). The required amount of loss is not constant and is a function of crystal temperature, pump power, spectral characteristics of cavity optics, and might vary with time during laser operation. Hence, even though one can achieve two-color laser operation for example by using a specially designed output coupler that equalizes the gain for a specific set of parameters, the intensities of lasing transitions will not be immune to fluctuations in external factors. On the other hand, usage of an off-surface optical axis BRF enables creation of a filter with variable parameters. Altering the filter parameters requires adjustment of the filters rotation angle only (about an axis normal to the surface), and this could be used for long-term stable multicolor laser operation with a suitable feedback mechanism.

To provide a little more insight into the advantages of such BRFs, we need to look at their working mechanism shortly. In a nutshell, a birefringent filter inside a laser cavity will transform the incident, let us assume TM-polarized, light into some elliptical polarization that will contain both TM and TE modes. For the linear cavity we have used in this study (Fig. 1), the Brewster surfaces of the laser crystal and the birefringent plate will create around 70\% Fresnel reflection loss in one round trip for the TE-polarized part of the beam (this is the maximum modulation depth that can be achieved from the filter). This loss, which is a consequence of the change in polarization state, depends on many parameters, including wavelength, thickness of the birefringent plate, the rotation angle of the birefringent filter, as well as the angle between the optical axis and the surface normal of the plate [39,42-49]. Achieving the required filtering characteristics from the BRF for a specific application requires careful selection of filter material, its thickness, and optic axis diving angle (angle between the surface of the plate and the optical axis).

As an example, Fig. 3 shows the calculated transmission characteristics of our linear Cr:Nd:GSGG laser cavity (Fig. 1) around the wavelength region of interest for one round trip (employing a 3-mm-thick crystal quartz BRF with an optical axis pointing $45^{\circ}$ out of the surface). The calculation is based on computation of the eigenvalues of the round-trip Jones matrix and details can be found in [39,42-49]. The transmission is plotted for four different rotation angles of the birefringent plate in the range from $66.2^{\circ}$ to $114.5^{\circ}$. In Fig. 3, the rotation

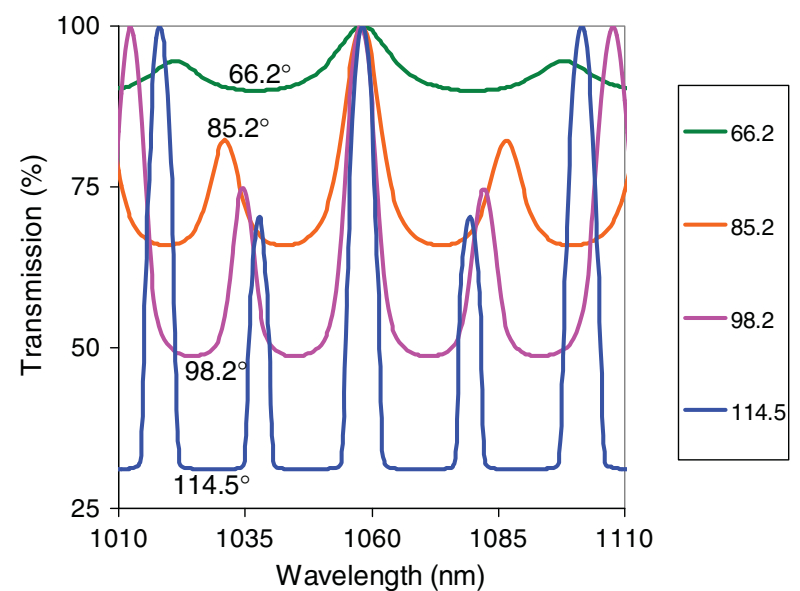

Fig. 3. Calculated transmission characteristics of the linear Cr:Nd:GSGG laser cavity (shown in Fig. 1) with the BRF, as a function of wavelength for different birefringent plate rotation angles (rotation angles in the range from $66.2^{\circ}$ to $114.5^{\circ}$; see differently colored graphs). The calculation has been performed for a 3-mm-thick crystal quartz birefringent plate with an optical axis tilted $45^{\circ}$ with respect to the surface of the plate.

angles are chosen such that the transmission at $1058 \mathrm{~nm}$ is maximized (there are in total 21 angles in the $0^{\circ}-180^{\circ}$ range that provide a $100 \%$ reflection at $1058 \mathrm{~nm}$, only four of them are plotted in Fig. 2 for simplicity). Note that, for the angles that we have chosen to plot, the modulation depth of the filter is also reasonably high (see Fig. 6(b) in [39]). As discussed earlier, let us assume we are trying to obtain simultaneous multicolor laser operation both at 1058 and $1061 \mathrm{~nm}$. As it is apparent from Fig. 3, since we have used an off-surface optical axis birefringent filter, each order of the filter has quite different filter parameters (modulation depth, FWHM, free spectral range, tuning speed, etc.). From Fig. 3, we see that, by adjusting the filter's rotation angle, we can create round-trip loss values ranging from $0 \%$ to $30 \%$ for the gain peak at $1061 \mathrm{~nm}$ while maintaining the transmission at $1058 \mathrm{~nm}$ at $100 \%$, and this design flexibility enables fine tuning of intensities in multicolor laser operation. In the experiments, we have tried different orders of the BRF by adjusting its rotation angle and we have chosen the rotation angle which enabled multicolor laser operation at these transitions with the best stability and power tuning capability (here order refers to angles where there is a separate transmission maxima). Note that in a regular birefringent filter with an optical axis lying on the surface of the plate, filter parameters only vary slightly as the filter is rotated and such BRFs do not possess all the advantages that we have just summarized, but they could still provide robust multicolor laser operation [39,42-49].

\section{B. Continuous-Wave Lasing Results}

Figure 4 shows the measured variation of Cr:Nd:GSGG lasers output power with absorbed pump power for the single-mode diode-pumped system. Six different output couplers with transmissions ranging from $0.15 \%$ to $5.4 \%$ were used in the experiments. With the $5.4 \%$ transmitting output coupler, the Cr:Nd:GSGG laser produced $32 \mathrm{~mW}$ of output power at 


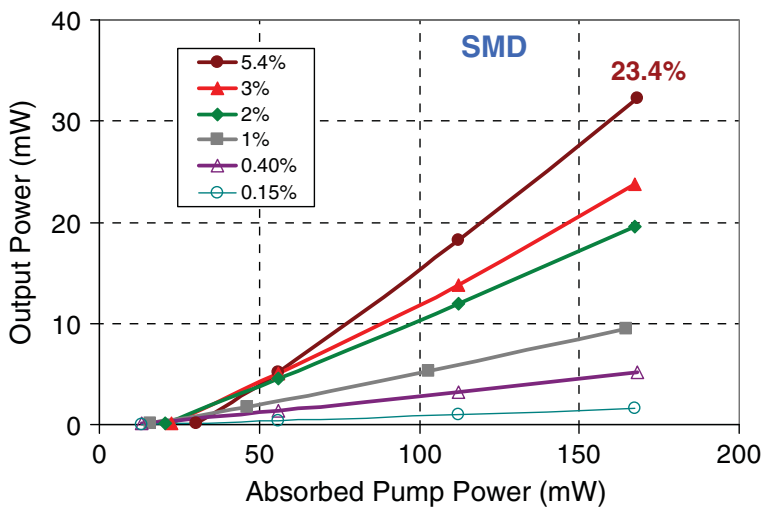

Fig. 4. Measured laser efficiency of single-mode diode (SMD) pumped Cr:Nd:GSGG laser using output couplers with different transmission values. Highest $\mathrm{cw}$ output power and slope efficiency attained were $32 \mathrm{~mW}$ and $23.4 \%$, respectively.

an absorbed pump power of $167 \mathrm{~mW}$. The lasing threshold and slope efficiency were $30 \mathrm{mw}$ and $23.4 \%$, respectively. A lasing threshold as low as $13 \mathrm{~mW}$ was measured using the $0.15 \%$ output coupler. The free-running laser wavelength was $1061 \mathrm{~nm}$. The laser crystal was not water cooled during the SMD pumping experiments.

Similarly, Fig. 5 shows the measured laser efficiency of the multimode diode-pumped Cr:Nd:GSGG laser. The best laser performance was again attained with the $5.4 \%$ transmitting output coupler. Laser output powers as high as $738 \mathrm{~mW}$ were obtained at an absorbed pump power of $4635 \mathrm{~mW}$. The corresponding lasing threshold and slope efficiency were $81 \mathrm{~mW}$ and $16 \%$, respectively. Lasing threshold as low as $42 \mathrm{~mW}$ was found with a $0.15 \%$ transmitting output coupler. Compared to SMD pumping, due to the non-optimum beam profile of the MMD diodes, the laser slope efficiency was lower. As an example, with the $5.4 \%$ transmitting output coupler, the laser slope efficiency with respect to the absorbed pump power decreased from $23.4 \%$ to $16.2 \%$. We also note here that part of the decrease observed in slope efficiency with multimode diode pumping was due to the increasing role of thermal effects.

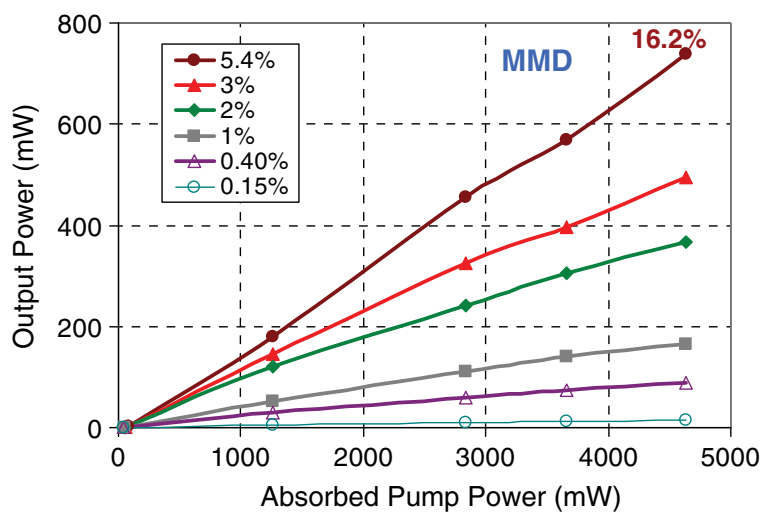

Fig. 5. Measured laser efficiency of multimode diode (MMD) pumped Cr:Nd:GSGG laser using output couplers with different transmission values. Highest $\mathrm{cw}$ output power and slope efficiency attained were $738 \mathrm{~mW}$ and $16.2 \%$, respectively.
To illustrate this issue, Fig. 6 shows the measured variation of the laser output power with crystal holder temperature at an absorbed pump power of $4635 \mathrm{~mW}$. Data are taken with three different output couplers. As is evident from Fig. 6, for all the output couplers, the obtainable laser powers decrease gradually with increasing temperature. Moreover, during the experiments, the Cr:Nd:GSGG laser required realignment at different incident pump power levels suggesting the role of pump-induced thermal lensing.

As indicated earlier, the free-running laser wavelength was $1061 \mathrm{~nm}$, which represents the maximum of the emission cross section for the Nd:GSGG material $[4,5,14]$. The abovementioned birefringent tuning plate was inserted into the cavity at Brewster's incidence to investigate cw single wavelength tuning capabilities of the laser. By adjusting the rotation angle of the birefringent filter, the laser could be operated at seven other transitions of the $\mathrm{Nd}$ ion. Figure 7 shows sample spectrum for each case, and also lists the obtainable output powers for each laser line. The output powers obtained for each line and the

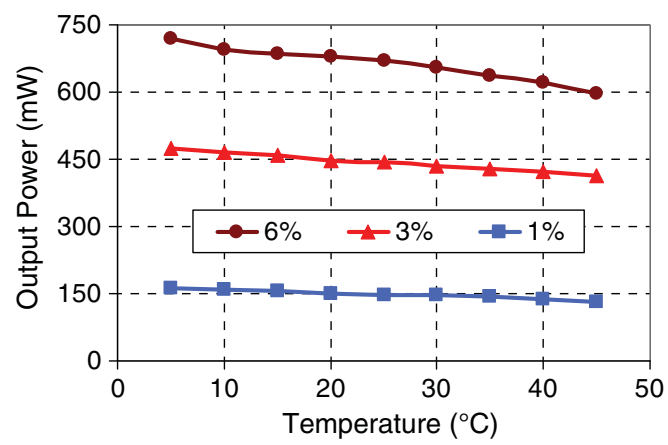

Fig. 6. Measured variation of laser output power with crystal holder temperature at $1 \%, 3 \%$, and $6 \%$ output coupling. The data was taken at an absorbed pump power of $4635 \mathrm{~mW}$.

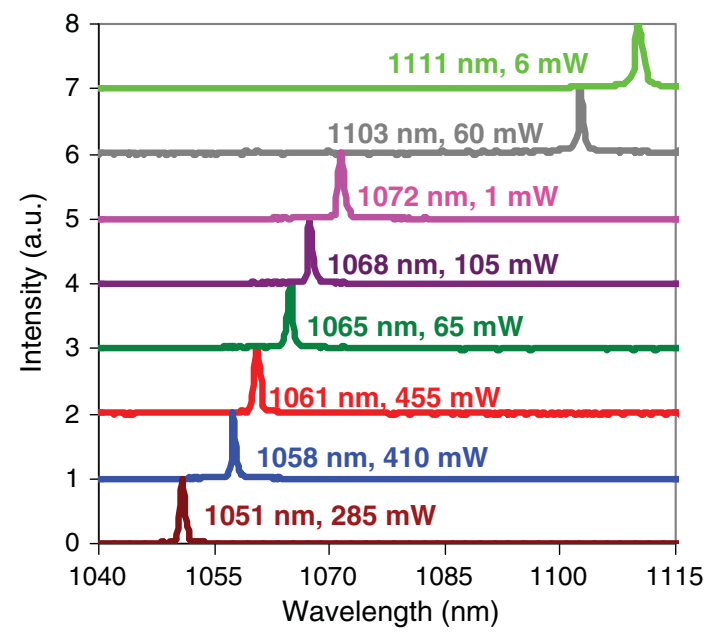

Fig. 7. Sample optical spectra of the Cr:Nd:GSGG laser during $\mathrm{cw}$ laser operation. The laser emission could be set to several different transitions of the $\mathrm{Nd}$ ion. The data was taken at an absorbed pump power of $4635 \mathrm{~mW}$. 
wavelength of the transitions are in good agreement with the measured strength of emission cross-section values for the different transitions of the Nd ion in GSGG (Fig. 2, and [5,15]).

Compared to earlier diode pumping results, the slope efficiencies obtained in this study are relatively low $(22.6 \%$ in [5,15] versus $16 \%$ in this study for MMD diode pumping). We suspected that this fact is due to the higher passive losses of the Cr:Nd:GSGG crystal that we have at hand during this study. To investigate this point, we have measured the intracavity laser resonator losses at the lasing wavelength $(\sim 1060 \mathrm{~nm})$ using two different approaches. In the first method, we have used Findlay-Clay analysis, which relates the measured lasing thresholds to output coupler transmission. It is very well known that the laser threshold pump power $\left(P_{\text {th }}\right)$ scales with the total round-trip loss $(L)$ of the cavity according to

$$
P_{\text {th }}=\frac{\pi\left(w_{p}^{2}+w_{c}^{2}\right) h \nu_{p}}{4\left(\sigma_{e}-\sigma_{\text {esa }}\right) \tau_{f} \eta_{p}}(T+L) .
$$

In Eq. (1) $h$ is Planck's constant, $\nu_{p}$ is the pump photon frequency, $\eta_{p}$ the pumping efficiency, $w_{c}\left(w_{p}\right)$ the cavity (pump) beam waists, $\sigma_{e}\left(\sigma_{\text {esa }}\right)$ the emission (excited-state absorption) cross section, $\tau_{f}$ the fluorescence lifetime of the upper laser level, and $T$ is the transmission of the output coupler. Figure 8 shows the measured variation of lasing threshold for the SMD and MMD-pumped Cr:Nd:GSGG laser using output couplers with different transmissions. Using Eq. (1), we have estimated the round-trip intracavity losses as $(4 \pm 1) \%$ and $(5.5 \pm 1) \%$ for the SMD and MMD-pumped systems, respectively.

As an alternative method, we have also exploited the measured variation of the laser slope efficiency $(\eta)$ as a function of output coupler transmission (Fig. 9) to estimate the level of passive cavity losses (Caird analysis [50,51]). In the Caird analysis, the slope efficiency $\eta$ of the laser can be estimated based on the following relationship:

$$
\eta=\left[\left(\frac{h v_{l}}{h v_{p}}\right) \eta_{p}\left(\frac{\sigma_{e}-\sigma_{\mathrm{ESA}}}{\sigma_{e}}\right)\right] \frac{T}{T+L},
$$

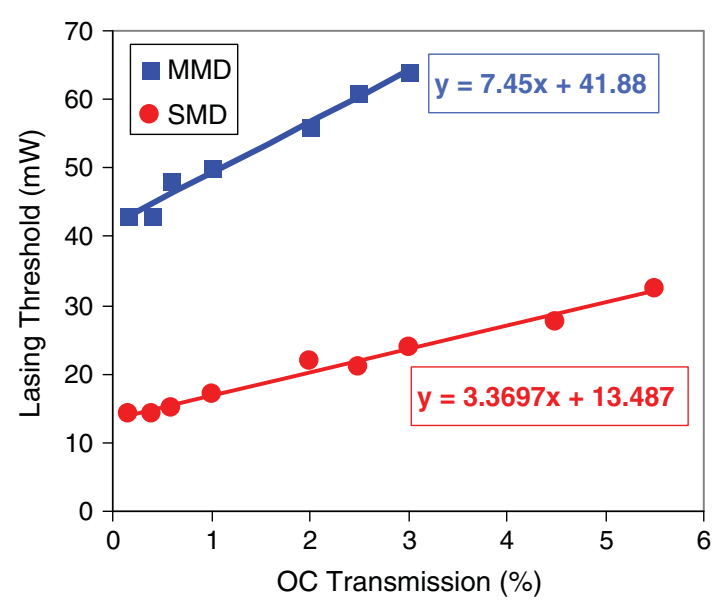

Fig. 8. Measured variation of lasing threshold $\left(P_{\text {th }}\right)$ with output coupler (OC) transmission $T$ for the single-mode diode (SMD) and multimode diode-pumped system. Using Findlay-Clay analysis, the round-trip passive cavity loss $L$ was estimated to be $(4 \pm 1) \%$ and $(5.5 \pm 1) \%$ for the SMD and MMD pumped systems, respectively.

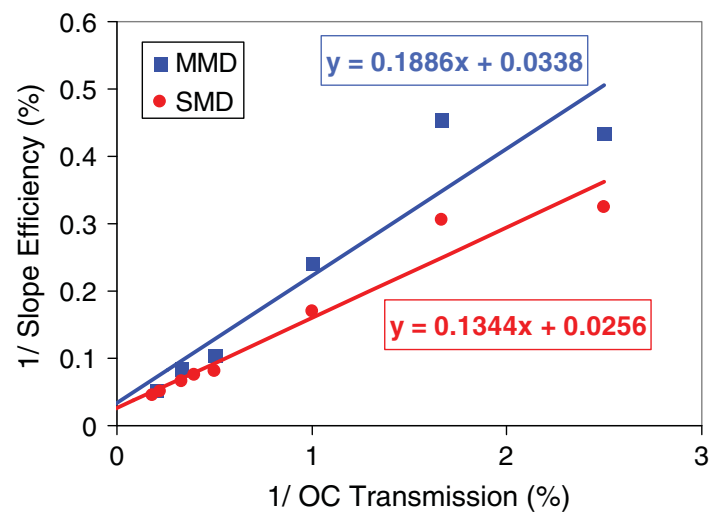

Fig. 9. Measured variation of the inverse of the slope efficiency $(1 / \eta)$ with the inverse of the output coupling percentage $(1 / T)$ for the single-mode diode (SMD) and multimode diode-pumped system. Employing Caird analysis, we have estimated the round-trip passive cavity loss $(L)$ to be $(5.3 \pm 1) \%$ and $(5.6 \pm 1) \%$, for the SMD and MMD pumped system, respectively.

where $\nu_{l}$ is the laser photon frequency, and the other terms are the same as in Eq. (1). Using Eq. (2), the best-fit values of $L$ were determined to be $(5.3 \pm 1) \%$ and $(5.6 \pm 1) \%$, for the SMD and MMD-pumped systems, respectively. Overall, both the Caird and Findlay-Clay analyses show that the round-trip loss of the cavity is around 5\%, which clearly deteriorated the laser performance we could get in this study. The corresponding passive loss of the crystal was estimated to be $2.3 \%$ per $\mathrm{cm}$ (compared to below $0.5 \%$ per $\mathrm{cm}$ in [52]). We believe that part of this high passive loss might be due to the presence of +4 charge state of the chromium ion, which has a known absorption band around $1 \mu \mathrm{m}$ [11]. Hence, future work with a Cr: Nd:GSGG crystal with lower passive losses could potentially improve the laser slope efficiencies obtained in this study.

\section{Mode-Locking Results}

\section{Mode-Locking at Single Wavelength}

Figure 10 shows the measured variation of laser output power and laser dynamics with absorbed pump power for the cavity with the SBR. The data is taken using the 1\% output coupler. The laser operated in the QSML regime for absorbed pump powers below $4 \mathrm{~W}$. For pump powers above $4 \mathrm{~W}$, stable CWML could be achieved. This behavior (transition from QSML to CWML) is quite well known in lasers mode-locked with an SBR [53]. Starting from rate equations for the intracavity laser power, gain and saturable absorption, one can show that in order to obtain stable $\mathrm{cw}$ mode-locking without $Q$-switching instabilities, the intracavity pulse energy should be above a critical value $\left(\mathrm{E}_{\mathrm{P}, \mathrm{c}}\right)$ [54-56]:

$$
\mathrm{E}_{\mathrm{P}, \mathrm{c}}=\sqrt{\frac{h \nu_{l}}{\left(m \sigma_{e}\right)} A_{\mathrm{eff}, L} F_{\mathrm{sat}, A} A_{\mathrm{eff}, A} \Delta \mathrm{R} .}
$$

In Eq. (3), $m$ is the number of passes through the laser crystal in one round trip, $A_{\text {eff, } L}\left(A_{\text {eff, } A}\right)$ is the effective laser mode area inside the gain medium (effective laser mode area on the absorber), $\mathrm{F}_{\mathrm{sar}, A}$ is the absorption saturation fluence of the SBR, and $\Delta \mathrm{R}$ is the modulation depth of the absorber. For the 


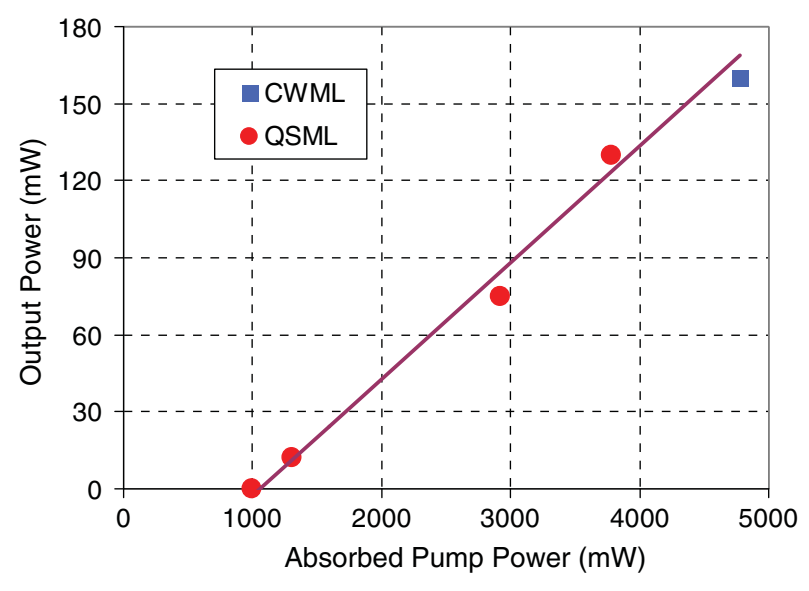

Fig. 10. Measured variation of laser output power with absorbed pump power for the cavity containing a saturable Bragg reflector (SBR) and a $1 \%$ output coupler. QSML, $Q$-switched mode-locking; CWML, continuous-wave mode-locking.

Cr:Nd:GSGG laser described in this work, we estimated the critical value of the intracavity pulse energy for stable $\mathrm{cw}$ modelocking $\left(\mathrm{E}_{\mathrm{P}, \mathrm{c}}\right)$ as $\sim 70 \mathrm{~nJ}\left(\mathrm{~m}=2, \sigma_{\mathrm{em}} \approx 3.1 \times 10^{-19} \mathrm{~cm}^{2}\right.$, $\mathrm{A}_{\mathrm{eff}, L} \approx \pi(30)(60) \mu \mathrm{m}^{2}, \quad \mathrm{~A}_{\mathrm{eff}, A} \approx \pi(20)(20) \mu \mathrm{m}^{2}, \quad \mathrm{~F}_{\mathrm{sar}, A} \approx$ $70 \mu \mathrm{J} / \mathrm{cm}^{2}$, and $\Delta \mathrm{R} \approx 0.6 \%$. From Fig. 10 , we see that for our system the experimentally realized value for $E_{P, c}$ is around $100 \mathrm{~nJ}$, which is quite close given the difficulty of determining the parameters used in Eq. (3) accurately.

Note that, once we were above the critical pulse energy, mode-locking was self-starting and it was immune to fluctuations. Figure 11 shows the autocorrelation traces taken at an absorbed pump power of $4.8 \mathrm{~W}$ (different colors represent data taken at different dates to confirm stability of pulse width in day to day operation). The laser produced 6 ps pulses (assuming sech $^{2}$ pulses) with $160 \mathrm{~mW}$ average power around $1061 \mathrm{~nm}$. The repetition rate was $142.65 \mathrm{MHz}$, resulting in pulse energies of $1.1 \mathrm{~nJ}$ and peak powers of $175 \mathrm{~W}$. The pulses also had a clean RF spectrum and the main peak was more than $55 \mathrm{dBm}$ above the noise floor (Fig. 12). Typical cw mode-locked laser

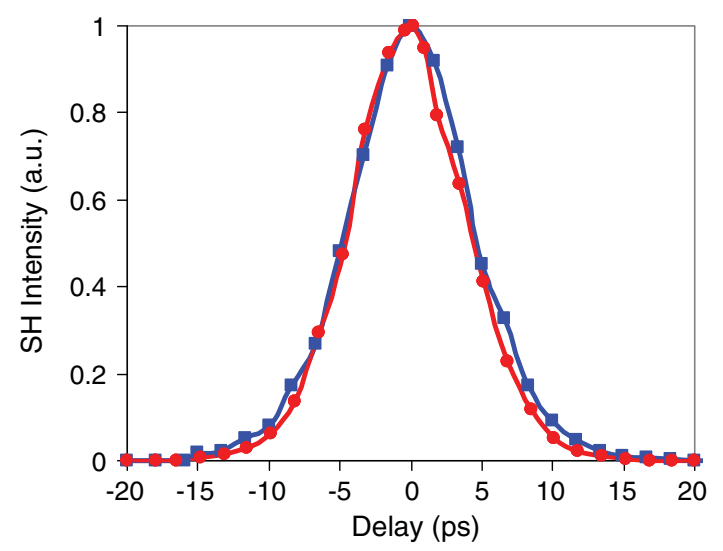

Fig. 11. Measured autocorrelation trace for the 6-ps-long pulses. The measurement performed at different days (indicated by different colors) resulted in similar pulse width measurements.

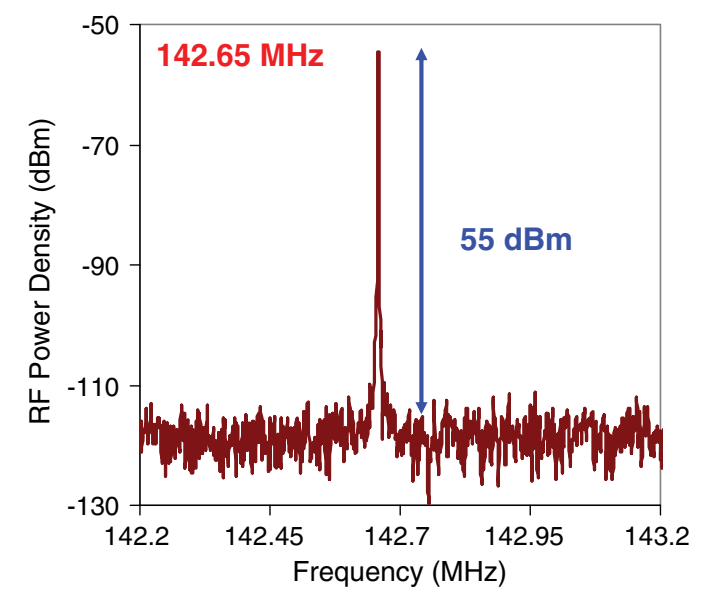

Fig. 12. Measured microwave spectrum indicating clean modelocking at $142.65 \mathrm{MHz}$ repetition rate.

pulse train is shown in Fig. 13. Note that, as we have seen in Fig. 5, optimum output coupling for the MMD-pumped laser system was around 5\%. On the other hand, in our modelocking studies, we have used a $1 \%$ output coupler to achieve clean $\mathrm{cW}$ mode-locked operation (to keep the intracavity pulse energies above $\mathrm{E}_{\mathrm{P}, \mathrm{c}}$ ). In future studies, using higher-power pump diodes and lower-loss laser crystals, one can potentially use higher output coupling for mode-locking, and this can improve the average powers obtained in this study significantly.

Earlier mode-locking studies with Cr:Nd:GSGG and Nd: GSGG have been performed in flashlamp-pumped systems where quasi-cw mode-locked operation has been obtained [54,55]. Passive mode-locking with dyes and active modelocking via acousto-optic modulators have been applied [57-60]. To our knowledge, this study presents the first demonstration of mode-locking in a diode-pumped system, and first use of SBR for mode-locking of Cr:Nd:GSSG and Nd: GSGG systems. The shortest pulses obtained so far with these materials is 4 ps long and achieved via passive mode-locking using dyes [57-60]. In our study the obtained pulse width (6 ps) was determined mostly by the SBR dynamics, since the cavity did not have negative dispersion. The bandwidth of the $1061 \mathrm{~nm}$ transition of Cr:GSGG is reported to be $11.5 \mathrm{~cm}^{-1}$ FWHM (around $1.3 \mathrm{~nm} ; 2.5$ times larger than $\mathrm{Nd}$ :YAG [57]); hence, future studies with optimally dispersion controlled cavities have the potential to reduce the pulse widths

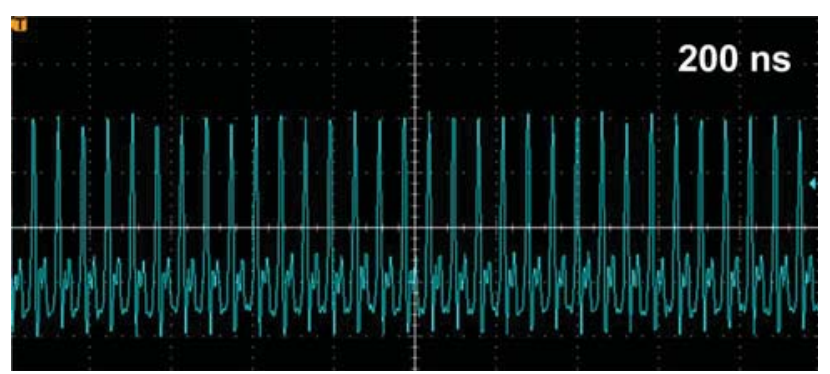

Fig. 13. Typical mode-locked pulse trains measured by a fast detector and an oscilloscope in nanosecond time scale. Total time window width is also indicated as a figure inset. 
to below 1.5 ps level (assuming transform-limited pulse width, a $1.3 \mathrm{~nm}$ FWHM around $1061 \mathrm{~nm}$ corresponds to $0.9 \mathrm{ps}$ and 1.25 ps long pulses for Sech ${ }^{2}$ and Gaussian-shaped spectrum, respectively).

\section{Simultaneous Mode-Locking at Multiple Wavelengths}

Using the off-surface optical axis birefringent plate, we have also tested tuning of the laser wavelength in the mode-locked regime. As it was noted earlier, with the $1 \%$ output coupler, the laser was just above the limit for $Q$-switched mode-locking (Fig. 10). Hence, we have used a $0.6 \%$ output coupler in our tuning studies to stay in the $\mathrm{cw}$ mode-locked regime as we tune the laser into emission lines with lower cross section. As shown in Fig. 14, by adjustment of the birefringent tuning plate's rotation angle, the wavelength of the mode-locked pulses could be selected as $1058 \mathrm{~nm}, 1061 \mathrm{~nm}$, or as their combination. The laser pulse width was around 6 ps for all cases. The laser operated in the $\mathrm{cw}$ mode-locked regime during tuning, and the measured average output power levels were indicated for each case as a figure inset. For the two-color mode-locked operation, it was possible to adjust the intensities of the laser lines by fine adjusting the rotation angle of the BRF. Note that the average power in two-color mode-locked operation (1058 and $1061 \mathrm{~nm}$ ) is only around $45 \mathrm{~mW}$ due to the usage of non-optimum output coupling $(0.6 \%)$ to suppress $Q$-switched mode-locking. As mentioned earlier, by using higher pump diodes it should be possible to scale the average powers obtained here considerably. For example, in two-color cw operation (1058 and $1061 \mathrm{~nm}$ ), we have obtained cw average powers of only $60 \mathrm{~mW}$ using the $0.6 \%$ transmitting output coupler, where as the $5.4 \%$ output coupler provided $390 \mathrm{~mW}$ of output. We finally note here that dual-wavelength modelocked operation could also be achieved for the 1051-1058 nm wavelength pair; however, the laser average power was even lower $(30 \mathrm{~mW})$, and the laser operated in the $Q$-switched mode-locked regime.

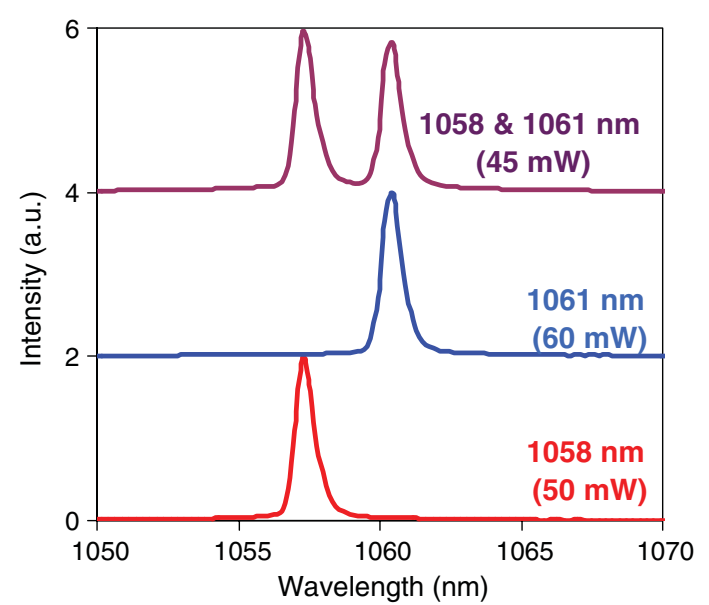

Fig. 14. Measured optical frequency spectrum for the dualwavelength $\mathrm{cw}$ mode-locked Cr:Nd:GSGG laser with the $0.6 \%$ output coupler. Mode-locking wavelength could be adjusted with the rotation of the birefringent tuning plate. Mode-locking at $1058 \mathrm{~nm}, 1061 \mathrm{~nm}$ or $1068 \mathrm{~nm}$ and $1061 \mathrm{~nm}$ could be chosen. The repetition rate was 155.3 MHz. Measured output power levels for each case are also indicated.

\section{CONCLUSION}

In summary, we have investigated cw and cw mode-locked laser performance of Cr:Nd:GSGG lasers pumped by state-of-theart reds single-mode and multimode diodes. In cw laser operation output powers as high as $738 \mathrm{~mW}$ were achieved around $1061 \mathrm{~nm}$. Cw lasing could also be attained at several other lines, including 1051, 1058, 1065, 1068, 1072, 1103, and $1111 \mathrm{~nm}$. When mode-locked with an SBR, the laser produced 6-ps-long pulses with a pulse energy of $1.1 \mathrm{~nJ}$ around $1061 \mathrm{~nm}$ at a repetition rate of $142.65 \mathrm{MHz}$. Using an off-surface optical axis birefringent filter, two-color picosecond laser operation was also achieved for the 1058-1061 nm and 1051-1058 nm transition pairs.

Funding. Türkiye Bilimsel ve Teknolojik Araştirma Kurumu (TÜBITAK) (114F191); H2020 European Research Council (ERC) (PCIG11GA-2012-321787).

Acknowledgment. We thank Golam Kibria from Antalya International University for his help in the early stage of the laser experiments. We would like to also thank the two anonymous reviewers, whose comments/suggestions helped improve and clarify this paper.

\section{REFERENCES}

1. E. Geusic, H. Marcos, and L. V. Uitert, "Laser oscillations in Nd-doped yttrium aluminum, yttrium gallium and gadolinium garnets," Appl. Phys. Lett. 4, 182-184 (1964).

2. S. E. Stokowski, "Nd:Cr:GSGG, will it replace Nd:YAG?" Proc. SPIE 0736, 22-28 (1987).

3. Z. J. Kiss and R. C. Duncan, "Cross-pumped $\mathrm{Cr}^{+3}-\mathrm{Nd}^{+3}$ :YAG laser system," Appl. Phys. Lett. 5, 200-202 (1964).

4. W. F. Krupke, M. D. Shinn, J. E. Marion, J. A. Caird, and S. E. Stokowski, "Spectroscopic, optical, and thermomechanical properties of neodymium- and chromium-doped gadalinium scandium gallium garnet," J. Opt. Soc. Am. B 3, 102-114 (1986).

5. A. A. Kaminskii, K. S. Bagdasarov, G. A. Bogomolova, M. M. Gritsenko, A. M. Kevorkov, and S. E. Sarkisov, "Luminescence and stimulated emission of $\mathrm{Nd}^{+3}$ ions in $\mathrm{Gd}_{3} \mathrm{Sc}_{2} \mathrm{Ga}_{3} \mathrm{O}_{12}$ crystals," Phys. Status Solidi A 34, K109-K114 (1976).

6. D. Pruss, G. Huber, and A. Beimowski, "Efficient $\mathrm{Cr}^{+3}$ sensitized $\mathrm{Nd}^{+3}$ : GdScGa-garnet laser at $1.06 \mu \mathrm{m}$," Appl. Phys. B 28, 355-358 (1982).

7. E. V. Zharikov, V. A. Zhitnyuk, G. M. Zverev, S. P. Kalitin, I. I. Kuratev, V. V. Laptev, A. M. Onishchenko, V. V. Osiko, V. A. Pashkov, A. S. Pimenov, A. M. Prokhorov, V. A. Smirnov, M. F. Stel'makh, A. V. Shestakov, and I. A. Shcherbakov, "Active media for high-efficiency neodymium lasers with nonselective pumping," Sov. J. Quantum Electron. 12, 1652-1653 (1982).

8. H. Hwang and J. H. Lee, "Efficiency and threshold pump intensity of cw solar-pumped solid-state lasers," IEEE J. Quantum Electron. 27, 2129-2134 (1991).

9. G. A. Thompson, V. Krupkin, A. Yogev, and M. Oron, "Solar-pumped Nd:Cr:GSGG parallel array laser," Opt. Eng. 31, 2644-2646 (1992).

10. M. Weksler and J. Shwartz, "Solar-pumped solid-state lasers," IEEE J. Quantum Electron. 24, 1222-1228 (1988).

11. R. Scheps, "Efficient $\mathrm{Cr}, \mathrm{Nd}: \mathrm{Gd}_{3} \mathrm{Sc}_{2} \mathrm{Ga}_{3} \mathrm{O}_{12}$ laser at $1.06 \mu \mathrm{m}$ pumped by visible GalnP/AIGalnP laser diodes," Appl. Phys. Lett. 59, 12871289 (1991)

12. D. P. Caffey and R. A. Utano, "Diode array side-pumped neodymiumdoped gadolinium scandium gallium garnet rod and slab lasers," Appl. Phys. Lett. 56, 808-810 (1990).

13. N. V. Kravtsov, O. E. Nanii, M. Y. Nikol'skii, I. A. Shcherbakov, V. V. Firsov, and E. V. Zharikov, "Unidirectional single-mode lasing on Nd-doped GSGG crystal," Opt. Mater. 1, 307-310 (1992). 
14. L. Tian, S. X. Wang, K. Wu, B. L. Wang, H. H. Yu, H. J. Zhang, H. Q. Cai, and $\mathrm{H}$. Huang, "Thermal, spectroscopic and laser properties of $\mathrm{Nd}^{3+}$ in gadolinium scandium gallium garnet crystal produced by optical floating zone method," Opt. Mater. 36, 521-528 (2013).

15. V. Lupei, N. Pavel, and A. Lupei, "Improved laser efficiency by direct diode laser pumping of the radiation-resistant $\mathrm{Nd}$ :gadoliniumscandium-gallium garnet," Laser Phys. 24, 045801 (2014).

16. M. Q. Wang, F. F. Zhang, J. J. Li, Z. M. Wang, N. Zong, S. J. Zhang, F. Yang, L. Yuan, Y. Bo, D. F. Cui, Q. J. Peng, and Z. Y. Xu, "Tunable millisecond narrow-band Nd:GSGG laser around $1336.6 \mathrm{~nm}$ for Al-27 (+) optical clock," Appl. Phys. B 122, 110 (2016).

17. E. V. Zharikov, V. V. Laptev, V. G. Ostroumov, Y. S. Privis, V. A. Smirnov, and I. A. Shcherbakov, "Investigation of a new laser active medium in the form of gadolinium scandium gallium garnet crystals activated with chromium and neodymium," Sov. J. Quantum Electron. 14, 1056-1062 (1984).

18. Z. Cong, D. Tang, W. T. De, J. Zhang, C. Xu, D. Luo, C. Xu, D. Li, J. $\mathrm{Xu}, \mathrm{X}$. Zhang, and Q. Wang, "Dual-wavelength passively mode-locked Nd:LuYSiO 5 laser with SESAM," Opt. Express 19, 3984-3989 (2011).

19. J. Hou, L. H. Zheng, J. L. He, J. Xu, B. T. Zhang, Z. W. Wang, F. Lou, R. H. Wang, and X. M. Liu, "A tri-wavelength synchronous modelocked Nd:SYSO laser with a semiconductor saturable absorber mirror," Laser Phys. Lett. 11, 035803 (2014).

20. A. Agnesi, F. Pirzio, G. Reali, A. Arcangeli, M. Tonelli, Z. Jia, and X. Tao, "Multi-wavelength diode-pumped Nd:LGGG picosecond laser," Appl. Phys. B 99, 135-140 (2010).

21. J. Hou, X. W. Fu, J. L. He, Y. Yang, B. T. Zhang, Z. W. Wang, K. J. Yang, Z. T. Jia, R. H. Wang, X. M. Liu, C. M. Dong, and X. T. Tao, "Dual-wavelength passively mode-locked Nd:LGGG laser with SESAM," IEEE Photon. Technol. Lett. 26, 40-42 (2014).

22. Y. Yang, Z. T. Jia, J. L. He, J. L. Xu, B. T. Zhang, R. H. Wang, X. M. Liu, J. Hou, F. Lou, Z. W. Wang, H. Yang, and X. T. Tao, "Dualwavelength synchronously passively mode-locked Nd:LGGG $1.3 \mu \mathrm{m}$ laser with SESAM," Laser Phys. Lett. 10, 045807 (2013).

23. G. Q. Xie, D. Y. Tang, H. Luo, H. J. Zhang, H. H. Yu, J. Y. Wang, X. T. Tao, M. H. Jiang, and L. J. Qian, "Dual-wavelength synchronously mode-locked Nd:CNGG laser," Opt. Lett. 33, 1872-1874 (2008).

24. J. L. Xu, S. Y. Guo, J. L. He, B. Y. Zhang, Y. Yang, H. Yang, and S. D. Liu, "Dual-wavelength asynchronous and synchronous mode-locking operation by a Nd:CLTGG disordered crystal," Appl. Phys. B 107, 53-58 (2012).

25. W. D. Tan, D. Y. Tang, C. W. Xu, J. Zhang, H. H. Yu, and H. J. Zhang, "Dual-wavelength passively mode-locked $\mathrm{Nd}$ : $\mathrm{GdVO}_{4}$ laser with orthogonal polarizations," Appl. Phys. B 102, 775-779 (2011).

26. Y.-J. Huang, T. Hsinchu, Y.-S. Tzeng, C.-Y. Tang, and Y.-F. Chen, "Efficient dual-wavelength synchronously mode-locked picosecond laser operating on the ${ }^{4} \mathrm{~F}_{3 / 2} \rightarrow{ }^{4} \mathrm{I}_{11 / 2}$ transition with compactly combined dual gain media," IEEE J. Sel. Top. Quantum Electron. 21, 354-360 (2015).

27. Y. J. Huang, Y. S. Tzeng, C. Y. Tang, S. Y. Chiang, H. C. Liang, and Y. F. Chen, "Efficient high-power terahertz beating in a dualwavelength synchronously mode-locked laser with dual gain media," Opt. Lett. 39, 1477-1480 (2014).

28. Y. J. Huang, H. H. Cho, Y. S. Tzeng, H. C. Liang, K. W. Su, and Y. F. Chen, "Efficient dual-wavelength diode-end-pumped laser with a diffusion-bonded $\mathrm{Nd}: \mathrm{YVO}_{4} / \mathrm{Nd}: \mathrm{GdVO}_{4}$ crystal," Opt. Mater. Express 5, 2136-2141 (2015).

29. A. Agnesi, A. Greborio, F. Pirzio, and G. Reali, "80-fs Nd: silicate glass laser pumped by a single-mode 200-mW diode," Opt. Express 18, 10098-10103 (2010).

30. Y. J. Huang, Y. S. Tzeng, H. H. Cho, and Y. F. Chen, "Effect of spatial hole burning on a dual-wavelength mode-locked laser based on compactly combined dual gain media," Photon. Res. 2, 161-167 (2014).

31. C. Feng, H. Zhang, Q. Wang, and J. Fang, "Dual-wavelength synchronously mode-locked laser of a $\mathrm{Nd}: \mathrm{Y}_{3} \mathrm{ScAl}_{4} \mathrm{O}_{12}$ disordered crystal," Laser Phys. Lett. 14, 045804 (2017).

32. T. L. Huang, C. L. Sung, H. P. Cheng, C. Y. Cho, H. C. Liang, K. W. Su, K. F. Huang, and Y. F. Chen, "Synchronized self-modelocked 1061-nm and 1064-nm monolithic Nd:YAG laser at cryogenic temperatures with two orthogonally polarized emissions: generation of 670 GHz beating," Opt. Express 24, 22189-22197 (2016).

33. C. L. Sung, C. Y. Lee, C. C. Chang, H. C. Liang, and Y. F. Chen, "Generation of terahertz optical beating from a simultaneously selfmode-locked Nd:YAG laser at 1064 and 1123 nm," Opt. Lett. 42, 302-305 (2017).

34. Y. J. Huang, Y. S. Tzeng, H. H. Cho, Y. F. Chen, W. D. Chen, G. Zhang, and T. C. Chen, "Generation of multiple spectral bands in a diode-pumped self-mode-locked Nd:YAP laser," Laser Phys. 26, 025803 (2016).

35. F. Zhang, X. W. Fan, J. Liu, F. K. Ma, D. P. Jiang, S. Y. Pang, L. B. Su, and J. Xu, "Dual-wavelength mode-locked operation on a novel $\mathrm{Nd}^{3+}, \mathrm{Gd}^{3+}: \mathrm{SrF}_{2}$ crystal laser," Opt. Mater. Express 6, 1513-1519 (2016).

36. C. G. Treviño-Palacios, C. Wetzel, and O. J. Zapata-Nava, "Design of a dual wavelength birefringent filter," AIP Conf. Proc. 992, 392-397 (2007).

37. C. G. Trevino-Palacios, O. J. Zapata-Nava, E. V. Mejia-Uriarte, N. Qureshi, G. Paz-Martinez, and O. Kolokolstev, "Dual wavelength continuous wave laser using a birefringent filter," J. Eur. Opt. Soc. Rapid Publ. 8, 13021 (2013).

38. R. Akbari, H. Zhao, and A. Major, "High-power continuous-wave dualwavelength operation of a diode-pumped Yb:KGW laser," Opt. Lett. 41, 1601-1604 (2016).

39. U. Demirbas, R. Uecker, J. G. Fujimoto, and A. Leitenstorfer, "Multicolor lasers using birefringent filters: experimental demonstration with Cr:Nd:GSGG and Cr:LiSAF," Opt. Express 25, 2594-2607 (2017).

40. S. Tsuda, W. H. Knox, S. T. Cundiff, W. Y. Jan, and J. E. Cunningham, "Mode-locking ultrafast solid-state lasers with saturable Bragg reflectors," IEEE Sel. Top. Quantum Electron. 2, 454-464 (1996).

41. U. Keller, K. J. Weingarten, F. X. Kärtner, D. Kopf, B. Braun, I. D. Jung, R. Fluck, C. Hönninger, N. Matuschek, and J. A. der Au, "Semiconductor saturable absorber mirrors (SESAM's) for femtosecond to nanosecond pulse generation in solid-state lasers," IEEE J. Sel. Top. Quantum Electron. 2, 435-453 (1996).

42. S. Lovold, P. F. Moulton, D. K. Killinger, and N. Menwk, "Frequency tuning characteristics of a Q-switched Co:MgF 2 Laser," IEEE J. Quantum Electron. 21, 202-208 (1985).

43. S. Zhu, "Birefringent filter with tilted optic axis for tuning dye lasers: theory and design," Appl. Opt. 29, 410-415 (1990).

44. A. L. Bloom, "Modes of a laser resonator containing tilted birefringent plates," J. Opt. Soc. Am. B 64, 447-452 (1974).

45. D. R. Preuss and J. L. Gole, "Three-stage birefringent filter tuning smoothly over the visible region: theoretical treatment and experimental design," Appl. Opt. 19, 702-710 (1980).

46. H. Wu, C. Zhang, and X. Bai, "A complete description of polarization and transmission of nonnormal incident rays in a uniaxial birefringent plate with arbitrary optic axis," Opt. Commun. 283, 4129-4134 (2010).

47. X. Wang and J. Yao, "Transmitted and tuning characteristics of birefringent filters," Appl. Opt. 31, 4505-4508 (1992).

48. K. Naganuma, G. Lenz, and E. P. Ippen, "Variable bandwidth birefringent filter for tunable femtosecond lasers," IEEE J. Quantum Electron. 28, 2142-2150 (1992).

49. B. Stormont, A. J. Kemp, I. G. Cormack, B. Agate, C. T. A. Brown, and W. Sibbett, "Broad tunability from a compact, low-threshold Cr:LiSAF laser incorporating an improved birefringent filter and multiplecavity Gires-Tournois interferometer mirrors," J. Opt. Soc. Am. B 22, 1236-1243 (2005).

50. D. Findlay and R. A. Clay, "The measurement of internal losses in 4-level lasers," Phys. Lett. 20, 277-278 (1966).

51. S. A. Payne, L. L. Chase, H. W. Newkirk, L. K. Smith, and W. F. Krupke, "LiCaAlF $: \mathrm{Cr}^{3+}$ a promising new solid-state laser material," IEEE J. Quantum Electron. 24, 2243-2252 (1988).

52. J. A. Caird, L. G. DeShazer, and J. Nella, "Characteristics of roomtemperature 2.3- $\mu \mathrm{m}$ laser emission from $\mathrm{Tm}^{3+}$ in $\mathrm{YAG}$ and $\mathrm{YAIO}_{3}$," IEEE J. Quantum Electron. 11, 874-881 (1975).

53. J. A. Caird, M. D. Shinn, T. A. Kirchoff, L. K. Smith, and R. E. Wilder, "Measurements of losses and lasing efficiency in GSGG:Cr, Nd and YAG:Nd laser rods," Appl. Opt. 25, 4294-4305 (1986). 
54. C. Hönninger, R. Paschotta, F. Morier-Genoud, M. Moser, and U. Keller, "Q-switching stability limits of continuous-wave passive mode locking," J. Opt. Soc. Am. B 16, 46-56 (1999).

55. F. X. Kärtner, L. R. Brovelli, D. Kopf, M. Kamp, I. Calasso, and U. Keller, "Control of solid-state laser dynamics by semiconductor devices," Opt. Eng. 34, 2024-2036 (1995).

56. U. Keller, "Recent developments in compact ultrafast lasers," Nature 424, 831-838 (2003).

57. A. V. Babushkin, N. S. Vorob'ev, E. V. Zharikov, S. P. Kalitin, V. V. Osiko, A. N. Prokhorov, Y. N. Serdyuchenko, M. Y. Shchelev, and I. A. Shcherbakov, "Picosecond laser made of a gadolinium scandium gallium garnet crystal doped with $\mathrm{Cr}$ and $\mathrm{Nd}$," Sov. J. Quantum Electron. 16, 428-429 (1986).

58. V. I. Prokhorenko, D. V. Surovtsev, E. A. Tikhonov, and D. Y. Yatskiv, "Stable pulsed picosecond GSGG: $\mathrm{Nd}^{3+}$ laser with a resonator based on Sagnac interferometer," Sov. J. Quantum Electron. 20, 228-231 (1990).

59. H. J. Eichler and B. Liu, "Picosecond Nd:Cr:GSGG-laser system with 30 mJ energy," Opt. Mater. 1, 21-25 (1992).

60. A. V. Kachinskii, I. V. Pilipovich, A. A. Stavrow, and N. A. Tylets, "Actively mode-locked GSGG:Cr:Nd lasers with negative feedback Q-control," J. Mod. Opt. 37, 1-4 (1990). 